\title{
Finite-Time $\mathrm{H}_{2} / \mathrm{H}_{\infty}$ Control for Linear Itô Stochastic Systems with $(x, u, v)$-Dependent Noise
}

\author{
Zhiguo Yan ${ }^{\mathbb{D}},{ }^{1,2,3}$ Shiyu Zhong, ${ }^{1}$ and Xingping Liu ${ }^{1}$ \\ ${ }^{1}$ School of Electrical Engineering and Automation, Qilu University of Technology (Shandong Academy of Sciences), \\ Jinan 250353, China \\ ${ }^{2}$ School of Control Science and Engineering, Shandong University, Jinan 250061, China \\ ${ }^{3}$ Key Laboratory of Pulp and Paper Science \& Technology of Ministry of Education of China, Qilu University of Technology \\ (Shandong Academy of Sciences), Jinan 250353, China \\ Correspondence should be addressed to Zhiguo Yan; yanzg500@sina.com
}

Received 2 January 2018; Revised 6 July 2018; Accepted 9 August 2018; Published 15 October 2018

Academic Editor: Michele Scarpiniti

Copyright (C) 2018 Zhiguo Yan et al. This is an open access article distributed under the Creative Commons Attribution License, which permits unrestricted use, distribution, and reproduction in any medium, provided the original work is properly cited.

\begin{abstract}
This paper deals with the problem of the $\mathrm{H}_{2} / \mathrm{H}_{\infty}$ control based on finite-time boundedness for linear stochastic systems. The motivation for investigating this problem comes from one observation that the $H_{2} / H_{\infty}$ control does not involve systems' transient performance. To express this problem clearly, a concept called finite-time $H_{2} / H_{\infty}$ control is introduced. Moreover, state feedback and observer-based finite-time $\mathrm{H}_{2} / \mathrm{H}_{\infty}$ controllers are designed, which guarantee finite-time boundedness, $\mathrm{H}_{2}$ performance index, and $H_{\infty}$ performance index of the closed-loop systems. Furthermore, an optimization algorithm on the finite-time $H_{2} / H_{\infty}$ control is presented to obtain the minimum values of the $H_{2}$ index and $H_{\infty}$ index. Finally, we use an example to show the validity of the obtained results.
\end{abstract}

\section{Introduction}

Recently, stochastic systems have been receiving more and more attention, which is due to their applications in many practical fields, such as finance systems [1] and power systems [2]. A lot of results on stochastic systems have been obtained. For example, [3] gave some Razumikhin-type theorems on the pth moment input-to-state stability for impulsive stochastic delayed systems by utilizing the Razumikhin technique and average dwell-time approach. By using the interval matrix transformation method, the problems of finite-time dissipative control for stochastic interval systems are investigated in [4]. The literature [5] investigated the state prediction problem for nonlinear stochastic differential systems by utilizing the Carleman embedding technique. In addition, many other nice results on stochastic systems have also been obtained; see, e.g., Lyapunov stability conditions [6], reliable output feedback control [7] and distributed containment control [8], and the references therein.
On the other hand, one of the important control methods in dealing with robust control problems is the $\mathrm{H}_{2} / \mathrm{H}_{\infty}$ control method. Its concrete implication is to seek a controller which not only minimizes the $\mathrm{H}_{2}$ cost function but also restrains the effect of external disturbance. The $\mathrm{H}_{2} / \mathrm{H}_{\mathrm{\infty}}$ control method has been applied to many fields, such as communication systems [9] and synthetic gene network design [10]. Meanwhile, $H_{2} / H_{\infty}$ control problems have been extended to many control system models, and many nice results have been obtained, such as stochastic systems [11], descriptor systems [12, 13], Markovian jump systems [14], and the recent monograph [15]. Nevertheless, most of the results on $\mathrm{H}_{2} / \mathrm{H}_{\infty}$ control problems in existing literature are based on Lyapunov's asymptotic stability, which do not reflect the transient performance of the systems. In some cases, large transient performance has a negative effect on the practical systems. For example, in power systems, a large transient current cannot be permitted, because it can damage the system [16]. In order to describe the phenomenon precisely, finite- 
time (FT) stability was introduced in the literature [17-19]. In the sequel, considering external disturbance, [20] extended FT stability to FT boundedness. Currently, the issues on FT stability and FT boundedness have been extensively studied for many kinds of system models; see, e.g. [21-30] and the references therein. Taking into account the advantages of FT stability and $\mathrm{H}_{2} / \mathrm{H}_{\infty}$ control, the finite-time $\mathrm{H}_{2} / \mathrm{H}_{\infty}$ control problem is proposed in this paper, which not only guarantees FTB but also satisfies the $H_{2} / H_{\infty}$ performance indices. Up to date, there is almost no literature to consider the FT $\mathrm{H}_{2} / \mathrm{H}_{\infty}$ control of stochastic systems.

This paper will study the problems of FT $\mathrm{H}_{2} / \mathrm{H}_{\infty}$ control for linear Itô stochastic systems. Because of the complexity of the problems, the appropriate controller design will be more difficult. By using the method of stochastic analysis, state feedback and observer-based FT $\mathrm{H}_{2} / \mathrm{H}_{\infty}$ controllers are designed. The main contributions of this paper are as follows: (i) The definition of the FT $H_{2} / H_{\infty}$ control for linear stochastic systems with $(x, u, v)$-dependent noise (which is called state-, control-, and disturbance-dependent noise) is first given, which simultaneously presents FT boundedness, $H_{2}$ performance index, and $H_{\infty}$ performance index, respectively, of the closed-loop system. (ii) The two new sufficient conditions for the existence of the state feedback controller and observer-based controller are obtained in the form of linear matrix inequalities (LMIs). (iii) A parameter optimization algorithm is given to obtain the minimum values of the $H_{2}$ performance index and $H_{\infty}$ performance index, simultaneously.

The organization of this paper is summarized as follows: In Section 2, we give some preliminaries. In Section 3, the state feedback FT $\mathrm{H}_{2} / \mathrm{H}_{\infty}$ controller is designed. In Section 4 , the observer-based FT $H_{2} / H_{\infty}$ controller is designed. In Section 5 , an optimization algorithm is provided for obtaining the minimum values of the $H_{2}$ performance index and $H_{\infty}$ performance index, simultaneously. In Section 6, a numerical example is discussed, and some remarks are concluded in Section 7.

Notation. $\left(\Omega, \mathscr{F},\left\{\mathscr{F}_{t}\right\}_{t \geq 0}, \mathbb{P}\right)$ : a complete probability space with a filtration $\left\{\mathscr{F}_{t}\right\}_{t \geq 0} . M^{\prime}$ : transpose of a matrix $M . M>$ $0: M$ is a positive definite symmetric matrix. $I_{n \times n}: n \times n$ identity matrix. $\mathscr{L}_{\mathscr{F}}^{2}\left(\mathscr{R}^{+}, \mathscr{R}^{l}\right)$ : the space of nonanticipative stochastic process $\xi(t)$ with respect to filtration $\mathscr{F}_{t}$ satisfying $\|\xi(t)\|_{L_{2}}^{2}:=E \int_{0}^{\infty}\|\xi(t)\|^{2} d t<\infty . \lambda_{\max }(M)$ and $\lambda_{\min }(M)$ : the maximum and minimum eigenvalue of matrix $M . E[\cdot]$ is the mathematical expectation of the stochastic process. The asterisk “ $*$ " in a matrix stands for the symmetry term. diag $\{\cdots\}$ represents a diagonal matrix. The "wrt" denotes "with respect to."

\section{Preliminaries}

We consider the linear stochastic system as follows:

$$
\left\{\begin{array}{l}
d x(t)=\left[A_{1} x(t)+B_{1} u(t)+G_{1} v(t)\right] d t+\left[A_{2} x(t)+B_{2} u(t)+G_{2} v(t)\right] d w(t), \\
y(t)=C_{1} x(t) \\
z(t)=C_{2} x(t)+D_{1} u(t)+D_{2} v(t), \\
x(0)=x_{0} \in R^{n}
\end{array}\right.
$$

where $A_{1}, A_{2}, B_{1}, B_{2}, C_{1}, C_{2}, D_{1}, D_{2}, G_{1}$, and $G_{2}$ are constant matrices with appropriate dimensions. $x(t) \in R^{n}, u(t) \in R^{m}$, $v(t) \in R^{p}, y(t) \in R^{q}$, and $z(t) \in R^{l}$ are called state, control input, external disturbance, measurement output, and controlled output, respectively. $x_{0}$ is the initial state. $w(t)$ is a one-dimensional Wiener process, which is defined as $(\Omega$, $\left.\mathscr{F},\left\{\mathscr{F}_{t}\right\}_{t \geq 0}, \mathbb{P}\right) . v(t)$ is the disturbance and satisfies the following set:

$$
\Omega=\left\{v(t): E \int_{0}^{t} v^{\prime}(s) v(s) d s<h, h>0, t \in[0, T]\right\} .
$$

Next, the definition of FT stochastic boundedness for system (1) is introduced, which is the generalization of FT boundedness in [20,31-33].
Definition 1. System (1) with $u(t)=0$ is FT stochastically bounded wrt $\left(d_{1}, d_{2}, T, R, h\right)$, if

$x^{\prime}(0) R x(0) \leq d_{1} \Rightarrow E\left[x^{\prime}(t) R x(t)\right]<d_{2}, \quad \forall t \in[0, T], \forall v(t) \in \Omega$,

where $0<d_{1}<d_{2}, T>0$, and $R>0$.

For the subsequent analysis, the following lemmas are useful for the FT $\mathrm{H}_{2} / \mathrm{H}_{\infty}$ controller design.

Lemma 1 [27]. If $\rho(t)>0, f \geq 0$, and $g \geq 0$ satisfy

$$
\rho(t) \leq f+g \int_{0}^{t} \rho(s) d s, \quad t \in[0, T],
$$


then we obtain

$$
\rho(t) \leq f \exp (g t)
$$

Lemma 2 [34]. Let $V(t, x) \in C^{1,2}\left(R^{+}, R^{+} \times R^{n}\right)$ be a scalar function and $V(t, x)>0$. For the following stochastic system

$$
d x(t)=a(x) d t+b(x) d w(t)
$$

the Itô formula of $V(t, x)$ is given as follows:

$$
d V(t, x)=\mathscr{L} \mathscr{V}(t, x) d t+\frac{\partial \mathscr{V}^{\prime}(t, x)}{\partial x} b(x) d w(t)
$$

where

$$
\begin{aligned}
\mathscr{L} \mathscr{V}(t, x)= & \frac{\partial \mathscr{V}(t, x)}{\partial t}+\frac{\partial \mathscr{V}^{\prime}(t, x)}{\partial x} a(x) \\
& +\frac{1}{2} b^{\prime}(x) \frac{\partial^{2} \mathscr{V}(t, x)}{\partial x^{2}} b(x) .
\end{aligned}
$$

\section{State Feedback Finite-Time $\mathrm{H}_{2} / \mathrm{H}_{\infty}$ Controller Design}

This section gives a problem formulation of state-feedback finite-time (SFFT) $H_{2} / H_{\infty}$ control for system (1). And also, the two sufficient conditions are given for the existence of a SF controller.

Considering the following linear state-feedback (SF) controller

$$
u(t)=K x(t)
$$

where $K$ is the feedback gain matrix.

Substituting the controller (9) into system (1) leads to the closed-loop system

$$
\left\{\begin{array}{l}
d x(t)=\left[\tilde{A}_{1} x(t)+G_{1} v(t)\right] \mathrm{d} t+\left[\tilde{A}_{2} x(t)+G_{2} v(t)\right] d w(t) \\
y(t)=C_{1} x(t) \\
z(t)=\tilde{C} x(t)+D_{2} v(t) \\
x(0)=x_{0} \in R^{n}
\end{array}\right.
$$

where $\tilde{A}_{1}=A_{1}+B_{1} K, \tilde{A}_{2}=A_{2}+B_{2} K$, and $\tilde{C}=C_{2}+D_{1} K$.

Associated with system (1), the following cost function is provided:

$$
J_{s}(x(t), u(t))=E \int_{0}^{T}\left[x^{\prime}(t) P_{1} x(t)+u^{\prime}(t) P_{2} u(t)\right] d t
$$

where $P_{1}>0$ and $P_{2}>0$ are the given positive scalars or given weighting matrices.
Also, by substituting (9) into (11), the corresponding performance function (11) becomes

$$
J_{s}(x(t))=E \int_{0}^{T}\left[x^{\prime}(t) P_{1} x(t)+\mathrm{x}^{\prime}(t) K^{\prime} P_{2} K x(t)\right] d t
$$

For a given $\gamma>0$, under the condition of zero initial value, the discretional nonzero disturbance $v(t)$ and the control output $z(t)$ satisfy the following form:

$$
E \int_{0}^{T} z^{\prime}(t) z(t) d t<\gamma^{2} E \int_{0}^{T} v^{\prime}(t) v(t) d t
$$

Based on the above preparations, we are in a position to give the definition of the state-feedback finite-time $\mathrm{H}_{2}$ $/ H_{\infty}$ control.

Definition 2. For some given positive scalars $\gamma, d_{1}, d_{2}, T, R$, and $h$, considering system (1), cost function (11), and the inequality (13), if there exist a positive scalar $J_{s}^{*}$ and a SF controller (9) such that

(i) system (10) is FT stochastically bounded wrt $\left(d_{1}\right.$, $\left.d_{2}, T, R, h\right)$

(ii) $H_{2}$ cost function (12) satisfies $J_{s}(x(t)) \leq J_{s}^{*}$ under the condition of $v(t)=0$

(iii) for any nonzero disturbance $v(t)$, under the zero initial condition, the inequality (13) is satisfied

then (9) is a state-feedback finite-time $\mathrm{H}_{2} / \mathrm{H}_{\infty}$ controller of the stochastic system (1).

Remark 1. Definition 2 considers three aspects of actual systems: FT boundedness of systems' states, minimum performance cost, and ability to suppress interference of the closed-loop systems, which is more complex than only considering the problems of the $\mathrm{H}_{2}$ performance index or $H_{\infty}$ performance index. In addition, these three aspects involved by the FT $H_{2} / H_{\infty}$ control are often required by the actual systems. For example, in the power systems, a large transient current cannot be permitted, electric energy consumption is expected to be minimum, and the system is expected to have better ability to suppress interference [16].

A sufficient condition for the existence of the SFFT $\mathrm{H}_{2} /$ $H_{\infty}$ controller will be given by the following theorem.

Theorem 1. For the given positive scalars $\gamma, d_{1}, d_{2}, T, R$, and $h$, if there exist a matrix $M>0$, a matrix $K$, and a scalar $\alpha \geq 0$ such that

$$
\left[\begin{array}{ccc}
\mathscr{T}_{1} & G_{1}+\tilde{M} \tilde{C}^{\prime} D_{2} & \tilde{M} \tilde{A}_{2}^{\prime} \\
* & -\gamma^{2} I+D_{2}^{\prime} D_{2} & G_{2}^{\prime} \\
* & * & -\tilde{M}
\end{array}\right]<0
$$




$$
\begin{aligned}
& {\left[\begin{array}{cc}
\mathscr{T}_{2} & \tilde{M} \tilde{A}_{2}^{\prime} \\
* & -\tilde{M}
\end{array}\right]<0,} \\
& \frac{d_{1}}{\lambda_{\min }(M)}+h \gamma^{2}<\frac{d_{2}}{\lambda_{\max }(M)} e^{-\alpha T},
\end{aligned}
$$

hold, where $\tilde{M}=R^{-1 / 2} M R^{-1 / 2}, \quad \mathscr{T}_{1}=\tilde{A}_{1} \tilde{M}+\tilde{M} \tilde{A}_{1}^{\prime}-\alpha \tilde{M}+$ $\tilde{M} \tilde{C}^{\prime} \tilde{C} \tilde{M}$, and $\mathscr{T}_{2}=\tilde{A}_{1} \tilde{M}+\tilde{M} \tilde{A}_{1}^{\prime}-\alpha \tilde{M}+\tilde{M} P_{1} \tilde{M}+\tilde{M} K^{\prime} P_{2}$ $K \tilde{M}$, then $u(t)=K x(t)$ is a SFFT $H_{2} / H_{\infty}$ controller of system (1) and the upper bound of the $H_{2}$ index can be given as $J_{\text {state }}^{*}=\lambda_{\max }\left(M^{-1}\right) d_{1} e^{\alpha T}$.

Proof. The proof will be divided into three steps.

Step 1. Prove that the condition (i) is satisfied.

It is noticed that

$\left[\begin{array}{ccc}\tilde{M} \tilde{C}^{\prime} \tilde{C} \tilde{M} & \tilde{M} \tilde{C}^{\prime} D_{2} & 0 \\ D_{2}^{\prime} \tilde{C} \tilde{M} & D_{2}^{\prime} D_{2} & 0 \\ 0 & 0 & 0\end{array}\right]=\left[\begin{array}{c}\tilde{M} \tilde{C}^{\prime} \\ D_{2}^{\prime} \\ 0\end{array}\right]\left[\begin{array}{c}\tilde{M} \tilde{C}^{\prime} \\ D_{2}^{\prime} \\ 0\end{array}\right]^{\prime} \geq 0$

Therefore, the condition (14) implies that

$$
\left[\begin{array}{ccc}
\tilde{A}_{1} \tilde{M}+\tilde{M} \tilde{A}_{1}^{\prime}-\alpha \tilde{M} & G_{1} & \tilde{M} \tilde{A}_{2}^{\prime} \\
* & -\gamma^{2} I & G_{2}^{\prime} \\
* & * & -\tilde{M}
\end{array}\right]<0 .
$$

Let $\mathscr{V}(x(t))=x^{\prime}(t) \tilde{M}^{-1} x(t)$, the infinitesimal operator $\mathscr{L}_{1} \mathscr{V}(x(t))$ of system (10) is defined as follows:

$$
\begin{aligned}
\mathscr{L}_{1} \mathscr{V}(x(t))= & {\left[\left(A_{1}+B_{1} K\right) x(t)+G_{1} v(t)\right]^{\prime} \tilde{M}^{-1} x(t) } \\
& +x^{\prime}(t) \tilde{M}^{-1}\left[\left(A_{1}+B_{1} K\right) x(t)+G_{1} v(t)\right] \\
& +\left[\left(A_{2}+B_{2} K\right) x(t)+G_{2} v(t)\right]^{\prime} \tilde{M}^{-1} \\
& \cdot\left[\left(A_{2}+B_{2} K\right) x(t)+G_{2} v(t)\right] \\
= & {\left[\begin{array}{l}
x(t) \\
v(t)
\end{array}\right]^{\prime}\left[\begin{array}{cc}
\bar{Z}_{1} & \tilde{A}_{1}^{\prime} \tilde{M}^{-1} G_{2}+\tilde{M}^{-1} G_{1} \\
* & G_{2}^{\prime} \tilde{M}^{-1} G_{2}
\end{array}\right]\left[\begin{array}{c}
x(t) \\
v(t)
\end{array}\right], }
\end{aligned}
$$

where $\bar{Z}_{1}=\tilde{A}_{1}^{\prime} \tilde{M}^{-1}+\tilde{M}^{-1} \tilde{A}_{1}+\tilde{A}_{2}^{\prime} \tilde{M}^{-1} \tilde{A}_{2}$.

Pre- and postmultiplying the inequality (18) by $\operatorname{diag}\left\{\tilde{M}^{-1}, I, \tilde{M}^{-1}\right\}$, it leads to the following inequality:

$$
\left[\begin{array}{ccc}
\tilde{A}_{1}^{\prime} \tilde{M}^{-1}+\tilde{M}^{-1} \tilde{A}_{1}-\alpha \tilde{M}^{-1} & \tilde{M}^{-1} G_{1} & \tilde{A}_{2}^{\prime} \tilde{M}^{-1} \\
* & -\gamma^{2} I & G_{2}^{\prime} \tilde{M}^{-1} \\
* & * & -\tilde{M}^{-1}
\end{array}\right]<0 .
$$

According to the Schur complement, (20) is equivalent to

$$
\left[\begin{array}{cc}
\tilde{A}_{1}^{\prime} \tilde{M}^{-1}+\tilde{M}^{-1} \tilde{A}_{1}+\tilde{A}_{2}^{\prime} \tilde{M}^{-1} \tilde{A}_{2}-\alpha \tilde{M}^{-1} & \tilde{M}^{-1} G_{1}+\tilde{A}_{2}^{\prime} \tilde{M}^{-1} G_{2} \\
* & G_{2}^{\prime} \tilde{M}^{-1} G_{2}-\gamma^{2} I
\end{array}\right]
$$

$<0$.

Considering the conditions (19) and (21), it follows that

$$
\mathscr{L}_{1} \mathscr{V}(x(t))<\alpha \mathscr{V}(x(t))+\gamma^{2} v^{\prime}(t) v(t)
$$

Integrating (22) from 0 to $t$ and then taking the expectation, it follows that

$$
\begin{aligned}
E \mathscr{V}(x(t))< & \mathscr{V}(x(0))+\alpha \int_{0}^{t} E \mathscr{V}(x(s)) d s \\
& +\gamma^{2} \int_{0}^{t} E v^{\prime}(s) v(s) d s .
\end{aligned}
$$

By Lemma 1, we get

$$
E \mathscr{V}(x(t))<\mathscr{V}(x(0)) e^{\alpha t}+\gamma^{2} e^{\alpha t} \int_{0}^{t} E v^{\prime}(s) v(s) d s
$$

According to known conditions, it yields

$$
\begin{aligned}
& E \mathscr{V}(x(t))=E\left[x^{\prime}(t) R^{1 / 2} M^{-1} R^{1 / 2} x(t)\right] \\
& \geq \lambda_{\min }\left(M^{-1}\right) E\left[x^{\prime}(t) R x(t)\right], \\
& \mathscr{V}(x(0)) e^{\alpha t}=x^{\prime}(0) R^{1 / 2} M^{-1 /} R^{12} x(0) e^{\alpha t} \\
& \leq \lambda_{\max }\left(M^{-1}\right) x^{\prime}(0) R x(0) e^{\alpha t} \\
& \leq \lambda_{\max }\left(M^{-1}\right) d_{1} e^{\alpha T} \\
& \gamma^{2} e^{\alpha t} \int_{0}^{t} E v^{\prime}(s) v(s) d s<e^{\alpha T} h \gamma^{2} .
\end{aligned}
$$

From (24), (25), (26), and (27), it is easy to obtain

$$
E\left[x^{\prime}(t) R x(t)\right]<\lambda_{\max }(M) e^{\alpha T}\left[\frac{d_{1}}{\lambda_{\min }(M)}+h \gamma^{2}\right] .
$$

According to condition (16), it follows that (28) leads to $\mathrm{E}\left[x^{\prime}(t) R x(t)\right]<d_{2}$ for all $t \in[0, T]$. So, 
the closed-loop system (10) is FT stochastically bounded wrt $\left(d_{1}, d_{2}, T, R, h\right)$.

Step 2. Prove that the condition (ii) is satisfied.

Under the condition of $v(t)=0$, the infinitesimal operator $\mathscr{L}_{2} \mathscr{V}(x(t))$ of system (10) is as follows:

$$
\begin{aligned}
\mathscr{L}_{2} \mathscr{V}(x(t))= & {\left[\tilde{A}_{1} x(t)\right]^{\prime} \tilde{M}^{-1} x(t)+x^{\prime}(t) \tilde{M}^{-1} \tilde{A}_{1} x(t) } \\
& +\left[\tilde{A}_{2} x(t)\right]^{\prime} \tilde{M}^{-1} \tilde{A}_{2} x(t) \\
= & x^{\prime}(t)\left[\tilde{A}_{1}^{\prime} \tilde{M}^{-1}+\tilde{M}^{-1} \tilde{A}_{1}+\tilde{A}_{2}^{\prime} \tilde{M}^{-1} \tilde{A}_{2}\right] x(t) .
\end{aligned}
$$

By Schur's complement, it can be seen that (15) is equivalent to

$$
\begin{aligned}
& \tilde{M} \tilde{A}_{1}^{\prime}+\tilde{A}_{1} \tilde{M}+\tilde{M} \tilde{A}_{2}^{\prime} \tilde{M}^{-1} \tilde{A}_{2} \tilde{M}+\tilde{M} P_{1} \tilde{M} \\
& \quad+\tilde{M} K^{\prime} P_{2} K \tilde{M}-\alpha \tilde{M}<0 .
\end{aligned}
$$

Premultiplying and postmultiplying (30) by $\tilde{M}^{-1}$, we obtain

$$
\begin{aligned}
& \tilde{A}_{1}^{\prime} \tilde{M}^{-1}+\tilde{M}^{-1} \tilde{A}_{1}+\tilde{A}_{2}^{\prime} \tilde{M}^{-1} \tilde{A}_{2}+P_{1} \\
& +K^{\prime} P_{2} K-\alpha \tilde{M}^{-1}<0 .
\end{aligned}
$$

According to (29) and (31), we get

$$
\begin{aligned}
& \mathscr{L}_{2} \mathscr{V}(x(t))-\alpha \mathscr{V}(x(t)) \\
& \quad+x^{\prime}(t)\left(P_{1}+K^{\prime} P_{2} K\right) x(t)<0 .
\end{aligned}
$$

Integrating (32) from 0 to $t, t \in[0, T]$, and taking the expectation, it is obtained that

$$
\begin{gathered}
E \int_{0}^{t} x^{\prime}(t)\left(P_{1}+K^{\prime} P_{2} K\right) x(t) d t+E \mathscr{V}(x(t)) \\
<\mathscr{V}(x(0))+\alpha E \int_{0}^{t} \mathscr{V}(x(t)) d t .
\end{gathered}
$$

From (33), we get

$$
\begin{gathered}
E \mathscr{V}(x(t))<\mathscr{V}(x(0))+\alpha E \int_{0}^{t} \mathscr{V}(x(t)) d t, \\
J_{s}(x(t))<\alpha E \int_{0}^{t} \mathscr{V}(x(t)) d t+\mathscr{V}(x(0)) .
\end{gathered}
$$

From (34), by Lemma 1, we obtain

$$
E \mathscr{V}(x(t))<\mathscr{V}(x(0)) e^{\alpha t}
$$

According to (35) and (36), it is obtained that

$$
\begin{aligned}
J_{s}(x(t)) & <\alpha E \int_{0}^{t} x^{\prime}(0) R^{1 / 2} M^{-1} R^{1 / 2} x(0) e^{\alpha t} d t+x^{\prime}(0) \tilde{M}^{-1} x(0) \\
& =x^{\prime}(0) R^{1 / 2} M^{-1} R^{1 / 2} x(0) e^{\alpha t}<\lambda_{\max }\left(M^{-1}\right) d_{1} e^{\alpha T}=J_{s}^{*} .
\end{aligned}
$$

Step 3. Prove that the condition (iii) is satisfied.

Premultiplying and postmultiplying (14) by diag $\left\{\tilde{M}^{-1}, I, \tilde{M}^{-1}\right\}$, and according to the Schur complement, we have

$$
\left[\begin{array}{cc}
\mathscr{T}_{3} & \tilde{A}^{\prime}{ }_{1} \tilde{M}^{-1} G_{2}+\tilde{M}^{-1} G_{1}+\tilde{C}^{\prime} D_{2} \\
* & -\gamma^{2} I+G_{2}^{\prime} \tilde{M}^{-1} G_{2}+D_{2}^{\prime} D_{2}
\end{array}\right]<0,
$$

where $\quad \mathscr{T}_{3}=\tilde{A}^{\prime} \tilde{M}^{-1}+\tilde{M}^{-1} \tilde{A}+\tilde{A}_{1}^{\prime} \tilde{M}^{-1} \tilde{A}_{1}-\alpha \tilde{M}^{-1}$ $+P_{1}+K^{\prime} P_{2} K+\tilde{C}^{\prime} \tilde{C}$.

According to (19) and (38), we have

$$
\mathscr{L}_{1} \mathscr{V}(x(t))<\alpha \mathscr{V}(x(t))+\gamma^{2} v^{\prime}(t) v(t)-z^{\prime}(t) z(t)
$$

Premultiplying and postmultiplying (39) by $e^{-\alpha t}$, we have

$$
\begin{aligned}
e^{-\alpha t} \mathscr{L}_{1} \mathscr{V}(x(t))< & \alpha e^{-\alpha t} \mathscr{V}(x(t)) \\
& +e^{-\alpha t}\left[\gamma^{2} v^{\prime}(t) v(t)-z^{\prime}(t) z(t)\right]
\end{aligned}
$$

By applying Lemma 2, we can obtain that

$$
\mathscr{L}_{1}\left[e^{-\alpha t} \mathscr{V}(x(t))\right]=-\alpha e^{-\alpha t} \mathscr{V}(x(t))+e^{-\alpha t} \mathscr{L}_{1} \mathscr{V}(x(t)) .
$$

According to (40) and (41), we get

$$
\mathscr{L}_{1}\left[e^{-\alpha t} \mathscr{V}(x(t))\right]<e^{-\alpha t}\left[\gamma^{2} v^{\prime}(t) v(t)-z^{\prime}(t) z(t)\right]
$$

Under the condition of zero initial value, integrating both sides of (42) from 0 to $t, t \in[0, T]$, taking the expectation, and after some calculations, we have

$$
\begin{aligned}
e^{-\alpha T} E \mathscr{V}(x(t)) & <e^{-\alpha T}\left[E \int_{0}^{T}\left(\gamma^{2} v^{\prime}(s) v(s) d s-z^{\prime}(s) z(s)\right) d s\right] \\
& <\gamma^{2} E \int_{0}^{T} v^{\prime}(s) v(s) d s-E \int_{0}^{t} z^{\prime}(s) z(s) d s .
\end{aligned}
$$




$$
\begin{aligned}
& \text { Because } e^{-\alpha T} E \mathscr{V}(x(t))>0 \text {, we can get } \\
& \qquad E \int_{0}^{T} z^{\prime}(s) z(s) d s<\gamma^{2} E \int_{0}^{T} v^{\prime}(s) v(s) d s .
\end{aligned}
$$

This completes the proof.

It should be noted that (14), (15), and (16) are not linear matrix inequalities and difficult to solve. To overcome the above difficulties, the following theorem is obtained.

Theorem 2. For the given positive scalars $\gamma, d_{1}, d_{2}, T, R$, and $h$, if there exist a matrix $M>0$, a matrix $Y$, a scalar $\lambda>0$, and a scalar $\alpha \geq 0$ such that

$$
\left[\begin{array}{cccc}
\mathscr{T}_{4} & G_{1} & \tilde{M} A_{2}^{\prime}+Y^{\prime} B_{2}^{\prime} & \tilde{M} C_{2}^{\prime}+Y^{\prime} D_{1} \\
* & -\gamma^{2} I & G_{2}^{\prime} & D_{2} \\
* & * & -\tilde{M} & 0 \\
* & * & * & -I
\end{array}\right]<0
$$

$$
\begin{array}{cccc}
{\left[\begin{array}{cccc}
\mathscr{T}_{4} & \tilde{M} A_{2}^{\prime}+Y^{\prime} B^{\prime}{ }_{2} & \tilde{M} & Y^{\prime} \\
* & -\tilde{M} & 0 & 0 \\
* & * & -P_{1}^{-1} & 0 \\
* & * & * & -P_{2}^{-1}
\end{array}\right]<0,} \\
& {\left[\begin{array}{ccc}
h \gamma^{2}-d_{2} e^{-\alpha T} & \sqrt{d_{1}} \\
* & & -\lambda
\end{array}\right]<0,} \\
& & & \lambda I<M<I,
\end{array}
$$

hold, where $\mathscr{T}_{4}=A_{1} \tilde{M}+\tilde{M} A_{1}^{\prime}-\alpha \tilde{M}+B_{1} Y+Y^{\prime} B_{1}^{\prime}$, then $u(t)=K x(t)=Y \tilde{M}^{-1} x(t)$ is a SFFT $H_{2} / H_{\infty}$ controller and the upper bound of the $\mathrm{H}_{2}$ index can be given as $J_{\text {state }}^{*}=\lambda^{-1}$ $d_{1} e^{\alpha T}$.

Proof. Letting $Y=K \tilde{M}$, (45) and (46) can lead to inequalities (14) and (15), respectively, and it is easy to check that (16) in Theorem 1 can be guaranteed by inequalities (47) and (48). The proof is completed.

Remark 2. It is noted that the inequalities in Theorem 2 are not LMIs, but if $\alpha$ is fixed, (45), (46), (47), and (48) can be regarded as LMIs.

\section{Observer-Based Finite-Time $H_{2} / H_{\infty}$ Controller Design}

In the above Section 3, the SFFT $H_{2} / H_{\infty}$ control problem of system (1) has been discussed. However, in many practical cases, it is difficult to measure the total states. Therefore, considering the problem of the observer-based finite-time (OBFT) $H_{2} / H_{\infty}$ controller design is necessary. Typically, an observer-based $(\mathrm{OB})$ controller is provided as follows:

$$
\left\{\begin{array}{l}
d \widehat{x}(t)=\left[A_{1} \widehat{x}(t)+B_{1} u(t)+L\left(y(t)-C_{1} \widehat{x}(t)\right)\right] d t, \\
u(t)=K \widehat{x}(t), \\
\widehat{x}(0)=0,
\end{array}\right.
$$

where $\hat{x}(t) \in \mathrm{R}^{n}$ is the estimation of $x(t)$ and $L$ is the estimator gain.

Substituting (49) into system (1), we will obtain the closed-loop system

$$
\left\{\begin{array}{l}
d \tilde{x}(t)=\left[\bar{A}_{1} \tilde{x}(t)+W_{1} v(t)\right] d t+\left[\bar{A}_{2} \tilde{x}(t)+W_{2} v(t)\right] d w(t) \\
\tilde{z}(t)=T_{1} \tilde{x}(t)+D_{2} v(t)
\end{array}\right.
$$

and the corresponding closed-loop cost function

$$
J_{o}(\tilde{x}(t))=E \int_{0}^{T} \tilde{x}^{\prime}(t) \mathscr{T} \tilde{x}(t) d t
$$

where

$$
\begin{aligned}
\tilde{x}(t) & =\left[\begin{array}{l}
x(t) \\
\widehat{x}(t)
\end{array}\right], \\
\bar{A}_{1} & =\left[\begin{array}{cc}
A_{1} & B_{1} K \\
L C_{1} & A_{1}+B_{1} K-L C_{1}
\end{array}\right], \\
W_{1} & =\left[\begin{array}{c}
G_{1} \\
0
\end{array}\right], \\
\bar{A}_{2} & =\left[\begin{array}{cc}
A_{2} & B_{2} K \\
0 & 0
\end{array}\right], \\
W_{2} & =\left[\begin{array}{c}
G_{2} \\
0
\end{array}\right], \\
T_{1} & =\left[\begin{array}{c}
C_{2}^{\prime} \\
K^{\prime} D_{1}^{\prime}
\end{array}\right], \\
\mathscr{T} & =\left[\begin{array}{cc}
P_{1} & 0 \\
0 & K^{\prime} P_{2} K
\end{array}\right] .
\end{aligned}
$$

For a given $\gamma>0$, under the condition of zero initial value, the discretional nonzero disturbance $v(t)$ and the control output $z(t)$ satisfy the following form:

$$
E \int_{0}^{T} \tilde{z}^{\prime}(t) \tilde{z}(t) d t<\gamma^{2} E \int_{0}^{T} v^{\prime}(t) v(t) d t
$$

Then, the definition of the observer-based finite-time $\mathrm{H}_{2} / \mathrm{H}_{\infty}$ control is given as follows.

Definition 3. For the given positive scalars $\gamma, d_{1}, d_{2}, T, R$, and $h$, considering system (1), cost function (11), and the 
inequality (13), if there exist an OB controller (49) and a positive scalar $J_{o}^{*}$ such that

(i) system (50) is FT stochastically bounded wrt $\left(d_{1}\right.$, $\left.d_{2}, T, R, h\right)$

(ii) $\mathrm{H}_{2}$ cost function (51) satisfies $J(\tilde{x}(t)) \leq J_{o}^{*}$ under the condition of $v(t)=0$

(iii) for any nonzero disturbance, under the zero initial condition, the inequality (54) is satisfied

then (49) is said to be an observer-based finite-time $H_{2} / H_{\infty}$ controller for the stochastic system (1).

Remark 3. From Definition 3, it can be seen that the existence of the observer-based finite-time $\mathrm{H}_{2} / \mathrm{H}_{\infty}$ controller (49) implies the existence of the SFFT $H_{2} / H_{\infty}$ controller.

By the results in Theorem 2, we have designed a statefeedback finite-time $\mathrm{H}_{2} / H_{\infty}$ controller $u(t)=K x(t)$. Next, a design condition of the observer-based finite-time $\mathrm{H}_{2} / \mathrm{H}_{\mathrm{\infty}}$ controller for the stochastic system (1) will be given.

Theorem 3. For the given positive scalars $\gamma, d_{1}, d_{2}, T, R$, and $h$, if there exist a positive matrix $Q$ and a scalar $\beta \geq 0$ such that

$$
\begin{gathered}
{\left[\begin{array}{ccc}
\mathscr{T}_{5} & \tilde{Q} W_{1}+T_{1}^{\prime} D_{2} & \bar{A}_{2}^{\prime} \tilde{Q} \\
* & -\gamma^{2} I+D_{2}^{\prime} D_{2} & W_{2}^{\prime} \tilde{Q} \\
* & * & -\tilde{Q}
\end{array}\right]<0,} \\
\bar{A}_{1}^{\prime} \tilde{Q}+\tilde{Q} \bar{A}_{1}+\bar{A}_{2}^{\prime} \tilde{Q} \bar{A}_{2}-\beta \tilde{Q}+\mathscr{T}<0, \\
\lambda_{\max }(Q) d_{1}+h \gamma^{2}<\lambda_{\min }(Q) d_{2} e^{-\beta T},
\end{gathered}
$$

hold, where $\tilde{Q}=R^{1 / 2} Q R^{1 / 2}$ and $\mathscr{T}_{5}=\bar{A}_{1}^{\prime} \tilde{Q}+\tilde{Q} \bar{A}_{1}-\beta \tilde{Q}+T_{1}$ $' T_{1}$, then (49) is an OBFT $H_{2} / H_{\infty}$ controller and the upper bound of the $\mathrm{H}_{2}$ index can be given as $J_{o}^{*}=\lambda_{\max }(Q) d_{1} e^{\beta T}$.

Proof. The proof will be divided into three steps.

Step 1. Prove that the condition (i) is satisfied.

Let $\mathscr{V}(\tilde{x}(t))=\tilde{x}^{\prime}(t) \tilde{Q} \tilde{x}(t)$ with $\tilde{Q}>0$ be solutions to (55), (56), and (57). The infinitesimal operator $\mathscr{L}_{3} \mathscr{V}(\tilde{x}(t))$ of system (50) is defined as follows:

$$
\begin{aligned}
\mathscr{L}_{3} \mathscr{V}(\tilde{x}(t))= & {\left[\bar{A}_{1} \tilde{x}(t)+W_{1} v(t)\right]^{\prime} \tilde{Q} \tilde{x}(t) } \\
& +\tilde{x}^{\prime}(t) \tilde{Q}\left[\bar{A}_{1} \tilde{x}(t)+W_{1} v(t)\right] \\
& +\left[\bar{A}_{2} \tilde{x}(t)+W_{2} v(t)\right]^{\prime} \tilde{Q}\left[\bar{A}_{2} \tilde{x}(t)+W_{2} v(t)\right]
\end{aligned}
$$

$$
\begin{aligned}
= & \tilde{x}^{\prime}(t)\left[\bar{A}_{1}^{\prime} \tilde{Q}+\tilde{Q}_{1}+\bar{A}_{2}^{\prime} \tilde{Q} \bar{A}_{2}\right] \tilde{x}(t) \\
& +v^{\prime}(t)\left[W_{1}^{\prime} \tilde{Q}+W_{2}^{\prime} \tilde{Q} \bar{A}_{2}\right] \tilde{x}(t) \\
& +\tilde{x}^{\prime}(t)\left[\tilde{Q} W_{1}+\bar{A}_{2}^{\prime} \tilde{Q} W_{2}\right] v(t) \\
& +v^{\prime}(t)\left[W_{2}^{\prime} \tilde{Q} W_{2}\right] v(t)=\left[\begin{array}{c}
\tilde{x}(t) \\
v(t)
\end{array}\right] \\
& +\left[\begin{array}{cc}
\bar{A}_{1}^{\prime} \tilde{Q}+\tilde{Q} \bar{A}_{1}+\bar{A}_{2}^{\prime} \tilde{Q} \bar{A}_{2} & \tilde{Q} W_{1}+\bar{A}_{2}^{\prime} \tilde{Q} W_{2} \\
* & W_{2}^{\prime} \tilde{Q} W_{2}
\end{array}\right] \\
& +\left[\begin{array}{c}
\tilde{x}(t) \\
v(t)
\end{array}\right] .
\end{aligned}
$$

Note that

$$
\left[\begin{array}{ccc}
T_{1}^{\prime} T_{1} & T_{1}^{\prime} D_{2} & 0 \\
D_{2}^{\prime} T_{1} & D_{2}^{\prime} D_{2} & 0 \\
0 & 0 & 0
\end{array}\right]=\left[\begin{array}{c}
T_{1}^{\prime} \\
D_{2}^{\prime} \\
0
\end{array}\right]\left[\begin{array}{c}
T_{1}^{\prime} \\
D_{2}^{\prime} \\
0
\end{array}\right]{ }^{\prime} \geq 0 .
$$

Therefore, the inequality (55) implies that

$$
\left[\begin{array}{ccc}
\bar{A}_{1}^{\prime} \tilde{Q}+\tilde{Q} \bar{A}_{1}-\beta \tilde{Q} & \tilde{Q} W_{1} & \bar{A}_{2}^{\prime} \tilde{Q} \\
* & -\gamma^{2} I & W_{2}^{\prime} \tilde{Q} \\
* & * & -\tilde{Q}
\end{array}\right]<0 .
$$

According to the Schur complement, (60) can be transformed into

$$
\left[\begin{array}{cc}
\bar{A}_{1}^{\prime} \tilde{Q}+\tilde{Q} \bar{A}_{1}+\bar{A}_{2}^{\prime} \tilde{Q} \bar{A}_{2}-\beta \tilde{Q} & \tilde{Q} W_{1}+\bar{A}_{2}^{\prime} \tilde{Q} W_{2} \\
* & -\gamma^{2} I+W_{2}^{\prime} \tilde{Q} W_{2}
\end{array}\right]<0 .
$$

According to (58) and (61), we have

$$
\mathscr{L}_{3} \mathscr{V}(\tilde{x}(t))<\beta \mathscr{V}(\tilde{x}(t))+\gamma^{2} v^{\prime}(t) v(t) .
$$

Integrating (62) from 0 to $t$ and then taking the expectation, it follows that

$$
\begin{aligned}
E \mathscr{V}(\tilde{x}(t))< & \mathscr{V}(\tilde{x}(0))+\beta \int_{0}^{t} E \mathscr{V}(\tilde{x}(s)) d s \\
& +\gamma^{2} \int_{0}^{t} E v^{\prime}(s) v(s) d s .
\end{aligned}
$$


According to Lemma 1, it is obtained that

$E \mathscr{V}(\tilde{x}(t))<\mathscr{V}(\tilde{x}(0)) e^{\beta t}+\gamma^{2} e^{\beta t} \int_{0}^{t} E v^{\prime}(s) v(s) d s$

According to known conditions, it yields that

$$
\begin{aligned}
& E \mathscr{V}(\tilde{x}(t))=E\left[\tilde{x}^{\prime}(t) R^{1 / 2} Q R^{1 / 2} \tilde{x}(t)\right] \\
& \geq \lambda_{\min }(Q) E\left[\tilde{x}^{\prime}(t) R \tilde{x}(t)\right], \\
& \mathscr{V}(\tilde{x}(0)) \mathrm{e}^{\beta t}=\tilde{x}^{\prime}(0) R^{1 / 2} Q R^{1 / 2} \tilde{x}(0) e^{\beta t} \\
& \leq \lambda_{\max }(Q) \tilde{x}^{\prime}(0) R \tilde{x}(0) e^{\beta t} \\
& \leq \lambda_{\max }(Q) d_{1} e^{\beta T}, \\
& \gamma^{2} e^{\beta t} \int_{0}^{t} E v^{\prime}(s) v(s) d s<e^{\beta T} h \gamma^{2} .
\end{aligned}
$$

From (64), (65), (66), and (67), it is easy to obtain that

$$
E\left[\tilde{x}^{\prime}(t) R \tilde{x}(t)\right]<\frac{\lambda_{\max }(Q) d_{1} e^{\beta T}+h \gamma^{2} e^{\beta T}}{\lambda_{\min }(Q)}
$$

According to condition (57), it follows that (68) leads to $E\left[\tilde{x}^{\prime}(t) R \tilde{x}(t)\right]<d_{2}$ for all $t \in[0, T]$. So, the closed-loop system (50) is FT stochastically bounded wrt $\left(d_{1}, d_{2}, T, R, h\right)$.

Step 2. Prove that the condition (ii) is satisfied.

Under the condition of $v(t)=0$, the infinitesimal operator $\mathscr{L}_{4} \mathscr{V}(\tilde{x}(t))$ of system (50) is defined as follows:

$$
\begin{aligned}
\mathscr{L}_{4} \mathscr{V}(\tilde{x}(t))= & {\left[\bar{A}_{1} \tilde{x}(t)\right]^{\prime} \tilde{Q} \tilde{x}(t)+\tilde{x}^{\prime}(t) \tilde{Q}\left[\bar{A}_{1} \tilde{x}(t)\right] } \\
& +\left[\bar{A}_{2} \tilde{x}(t)\right]^{\prime} \tilde{Q}\left[\bar{A}_{2} \tilde{x}(t)\right] \\
= & \tilde{x}^{\prime}(t)\left[\bar{A}_{1}^{\prime} \tilde{Q}+\tilde{Q} \bar{A}_{1}+\bar{A}_{2}^{\prime} \tilde{Q} \bar{A}_{2}\right] \tilde{x}(t) .
\end{aligned}
$$

According to (56), we get

$$
\mathscr{L}_{4} \mathscr{V}(\tilde{x}(t))-\beta \mathscr{V}(\tilde{x}(t))+\tilde{x}^{\prime}(t) \mathscr{T} \tilde{x}(t)<0 .
$$

Integrating (70) from 0 to $t$ and taking the expectation, the following results are obtained:

$$
\begin{aligned}
E \mathscr{V} & (\tilde{x}(t))+E \int_{0}^{t} \tilde{x}^{\prime}(t) \mathscr{T} \tilde{x}(t) d t \\
& <\mathscr{V}(\tilde{x}(0))+\beta E \int_{0}^{t} \mathscr{V}(\tilde{x}(t)) d t
\end{aligned}
$$

From (71), we have

$$
\begin{array}{r}
E \mathscr{V}(\tilde{x}(t))<\mathscr{V}(\tilde{x}(0))+\beta E \int_{0}^{t} \mathscr{V}(\tilde{x}(t)) d t \\
E \int_{0}^{t} \tilde{x}^{\prime}(t) \mathscr{T} \tilde{x}(t) d t<\mathscr{V}(\tilde{x}(0))+\beta E \int_{0}^{t} \mathscr{V}(\tilde{x}(t)) d t .
\end{array}
$$

From (72), by Lemma 1, we obtain

$$
E \mathscr{V}(\tilde{x}(t))<\mathscr{V}(\tilde{x}(0)) e^{\beta t}
$$

From (73) and (74), we have

$$
\begin{aligned}
J_{o}(\tilde{x}(t)) & <\beta E \int_{0}^{t} \mathscr{V}(\tilde{x}(t)) d t+\mathscr{V}(\tilde{x}(0)) \\
& <\beta E \int_{0}^{t} \mathscr{V}(\tilde{x}(0)) e^{\beta t} d t+\mathscr{V}(\tilde{x}(0)) \\
& =\mathscr{V}(\tilde{x}(0)) e^{\beta t}<\lambda_{\max }(Q) d_{1} e^{\beta T}=J_{o}^{*} .
\end{aligned}
$$

Step 3. Prove that the condition (iii) is satisfied.

According to the Schur complement, it can be seen that (55) is equivalent to

$$
\left[\begin{array}{cc}
\bar{A}_{1}^{\prime} \tilde{Q}+\tilde{Q} \bar{A}_{1}-\beta \tilde{Q}+T_{1}^{\prime} T_{1}+\bar{A}_{2}^{\prime} \tilde{Q} \bar{A}_{2} & \tilde{Q} W_{1}+T_{1}^{\prime} D_{2}+\bar{A}_{2}^{\prime} \tilde{Q} W_{2} \\
* & W_{2}^{\prime} \tilde{Q} W_{2}+D_{2}^{\prime} D_{2}-\gamma^{2} I
\end{array}\right]<0 .
$$

According to (58) and (76), we get

$$
\mathscr{L}_{3} \mathscr{V}(\tilde{x}(t))<\beta \mathscr{V}(\tilde{x}(t))+\gamma^{2} v^{\prime}(t) v(t)-\tilde{z}^{\prime}(t) \tilde{z}(t)
$$

Using the similar procedure to prove Step 3 in Theorem 3, we can obtain

$$
E \int_{0}^{T} \tilde{z}^{\prime}(s) \tilde{z}(s) d s<\gamma^{2} E \int_{0}^{T} v^{\prime}(s) v(s) d s
$$

This completes the proof.

We find that inequalities (55), (56), and (57) in Theorem 3 are not easy to solve, so the following theorem will transform (55), (56), and (57) into LMIs.

Theorem 4. For the given positive scalars $\gamma, d_{1}, d_{2}, T, R$, and $h$, if there exist a scalar $\beta>0$, two matrices $\tilde{Q}_{11}>0$ and $\tilde{Q}_{22}>0$, a matrix $N$, and a scalar $\eta>0$ such that

$$
\left[\begin{array}{ccc}
\Sigma_{11} & \Sigma_{12} & \Sigma_{13} \\
* & \Sigma_{22} & \Sigma_{23} \\
* & * & \Sigma_{33}
\end{array}\right]<0
$$




$$
\begin{gathered}
{\left[\begin{array}{cc}
\mathscr{E}_{11}-\beta \tilde{Q}_{11} & \mathscr{E}_{12} \\
* & \mathscr{E}_{22}-\beta \tilde{Q}_{22}
\end{array}\right]<0,} \\
e^{\beta T}\left(\eta d_{1}+h \gamma^{2}\right)-d_{2}<0, \\
I<\operatorname{diag}\left\{\tilde{Q}_{11}, \tilde{Q}_{22}\right\}<\eta I,
\end{gathered}
$$

hold, where $\Sigma_{11}=A_{1}^{\prime} \tilde{Q}_{11}+\tilde{Q}_{11} A_{1}+A_{2}^{\prime} \tilde{Q}_{11} A_{2}+C_{2}^{\prime} C_{2}-\beta \tilde{Q}_{11}$, $\Sigma_{12}=C_{1}^{\prime} N^{\prime}+\tilde{Q}_{11} B_{1} K+A_{2}^{\prime} \tilde{Q}_{11} B_{2} K+C_{2}^{\prime} D_{1} K, \quad \Sigma_{22}=\left(A_{1}+B_{1}\right.$ $K)^{\prime} \tilde{Q}_{22}+\tilde{Q}_{22}\left(A_{1}+B_{1} K\right)+K^{\prime} B_{2}^{\prime} \tilde{Q}_{11} B_{2} K-N C_{1}-C_{1}^{\prime} N^{\prime}+K^{\prime}$ $D_{1}^{\prime} \quad D_{1} K-\beta \tilde{Q}_{22}, \quad \Sigma_{13}=\tilde{Q}_{11} G_{1}+C_{2}^{\prime} D_{2}+A_{2}^{\prime} \tilde{Q}_{11} G_{2}, \quad \Sigma_{23}=K^{\prime}$ $D_{1}^{\prime} D_{2}+K^{\prime} B_{2}^{\prime} Q_{11} G_{2}, \quad \Sigma_{33}=G_{2}^{\prime} \tilde{Q}_{11} G_{2}+D_{2}^{\prime} D_{2}-\gamma^{2} I, \quad \mathscr{E}_{11}=A_{1}^{\prime}$ $\tilde{Q}_{11}+\tilde{Q}_{11} A_{1}+A_{2}^{\prime} \tilde{Q}_{11} A_{2}+P_{1}, \quad \mathscr{E}_{12}=C_{1}^{\prime} N^{\prime}+\tilde{Q}_{11} B_{1} K+A_{2}^{\prime} \tilde{Q}_{11}$ $B_{2} K$, and $\mathscr{E}_{22}=\left(A_{1}+B_{1} K\right)^{\prime} \tilde{Q}_{22}+\tilde{Q}_{22}\left(A_{1}+B_{1} K\right)+K^{\prime} B_{2}^{\prime} \tilde{Q}_{11}$ $B_{2} K-N C_{1}-C_{1}^{\prime} N^{\prime}+K^{\prime} P_{2} K$, then (49) is an OBFT $H_{2} / H_{\infty}$ controller and the upper bound of the $\mathrm{H}_{2}$ index can be given as $J_{o}^{*}=\eta d_{1} e^{\beta T}$. In this case, an appropriate estimator gain matrix is given by $L=\tilde{Q}_{22}^{-1} N$.

Proof. Setting $\tilde{Q}=\operatorname{diag}\left\{\tilde{Q}_{11}, \tilde{Q}_{22}\right\}$, substituting (52) and (53) into (55), and letting $N=\tilde{Q}_{22} L$, (79) can be obtained from (55). It is obvious that (80) can be obtained from (56). And then, (81) and (82) imply (57). The proof is completed.

\section{Algorithm}

In this section, an optimization algorithm on the FT $\mathrm{H}_{2} / \mathrm{H}_{\infty}$ control is presented. The following algorithm is given for Theorem 2. Similarly, the algorithm can be utilized in Theorem 4.

Analysis. By Theorem 2, let $J_{s}^{*}<\xi$, and then minimize $\xi$, subject to (45), (46), (47), and (48), $M>0$ and

$$
\frac{M e^{-\alpha T}}{d_{1}}-\xi^{-1} I>0,
$$

where $0<d_{1}<d_{2}, T>0, \alpha \geq 0$, and $\lambda>0$.

By analyzing (45), (46), (47), and (48) in Theorem 2, we find the following: if (45), (46), (47), and (48) have no feasible solutions at the case of $\alpha=0$, then (45), (46), (47), and (48) will have no feasible solutions for all $\alpha>0$. Therefore, we seek $\alpha$ that makes (45), (46), (47), and (48) have feasible solutions from $\alpha=0$ by linear searching. The detailed algorithm will be given as follows. One can use this algorithm to obtain the minimum value of $\xi$ and $\gamma^{2}$.

By running Algorithm 1, we get the following results:

(1) If $U(i)=$ empty, $F(i)=$ empty, and $H(i)=$ empty, then it shows that the above optimization problem (OP) is not solvable.

(2) If $U(i) \neq$ empty, $F(i) \neq$ empty, and $H(i) \neq$ empty, then it shows that the above optimization problem is solvable. Moreover, we can respectively plot the curves of $(U(i), F(i)),(U(i), H(i))$, and $(F(i)$,
$H(i))$. The three curves can help us choose a suitable finite-time $\mathrm{H}_{2} / \mathrm{H}_{\infty}$ controller.

(3) This algorithm can find the most suitable $\alpha$ to decrease the conservativeness of the conditions of Theorem 2.

\section{Examples}

This section provides an example to show the effectiveness of the obtained results. System (10) with parameters is as follows:

$$
\begin{aligned}
A_{1} & =\left[\begin{array}{cc}
-10.1 & -10.8 \\
10.7 & -10.2
\end{array}\right], \\
A_{2} & =\left[\begin{array}{ll}
-0.8 & 1 \\
0.4 & -1
\end{array}\right], \\
B_{1} & =\left[\begin{array}{ll}
-2.1 & 5 \\
-4.1 & 3
\end{array}\right], \\
B_{2} & =\left[\begin{array}{ll}
-0.8 & 1.2 \\
-1 & 1.5
\end{array}\right], \\
G_{1} & =\left[\begin{array}{ll}
-0.5 & 0.4 \\
-0.1 & -0.5
\end{array}\right], \\
G_{2} & =\left[\begin{array}{ll}
0.2 & -0.1 \\
0.4 & 0.5
\end{array}\right], \\
C_{2} & =\left[\begin{array}{ll}
-1.9 & -0.1 \\
1.5 & -2.5
\end{array}\right], \\
C_{1} & =\left[\begin{array}{ll}
-0.8 & -1.5 \\
1.1 & -3
\end{array}\right], \\
P_{1} & =\left[\begin{array}{cc}
4 & -3 \\
-3 & 4
\end{array}\right], \\
P_{2}= & {\left[\begin{array}{ll}
3 & 2 \\
2 & 3
\end{array}\right], }
\end{aligned}
$$

and $d_{1}=1, d_{2}=5, D_{1}=2, D_{2}=0.2, T=1, R=I$, and $h=0.5$.

6.1. SFFT $\mathrm{H}_{2} / \mathrm{H}_{\infty}$ Controller Design. By running Algorithm 1 , the relations of $\alpha$ and $\xi$ (Figure 1), $\alpha$ and $\gamma$ (Figure 2), and $\gamma$ and $\xi$ (Figure 3 ) are obtained. From Figure 1, it can be seen that the bigger the number of $\alpha$, the bigger $\xi$ is. Also, the minimum value of the $H_{2}$ performance index is $\xi=1$ when $\alpha=0$, and the maximum value of the $H_{2}$ performance index is $\xi=4.4817$ when $\alpha=1.5$. The range of $\alpha$ is $[0,1.5]$, and the range of $\xi$ is $[1,4.4817]$.

From Figure 2, it can be seen that the bigger the number of $\alpha$, the smaller $\gamma$ is. Also, the maximum value of $\gamma$ is 
Step 1: Given $d_{1}, d_{2}, T, R, h$, and a step size $d_{\alpha}$ for $\alpha$.

Step 2: Set $i=1$ and take $\alpha_{1}=0$.

Step 3: If $\alpha_{i}$ makes the following optimization problem

$$
\begin{aligned}
& \min _{\text {s.t. }(45),(46),(47),(48),(83), M>0} \xi \text {, } \\
& \min _{\text {s.t. }(45),(46),(47),(48),(83), M>0} \gamma^{2} \text {, }
\end{aligned}
$$

solvable, then store $\alpha_{i}$ into $U(i), \xi_{\min }$ into $F(i)$, and $\gamma_{\min }$ into $H(i)$ and let $\alpha_{i+1}=\alpha_{i}+d_{\alpha}$, loop.

Otherwise, go to Step 4.

Step 4: Exit.

Algorithm 1

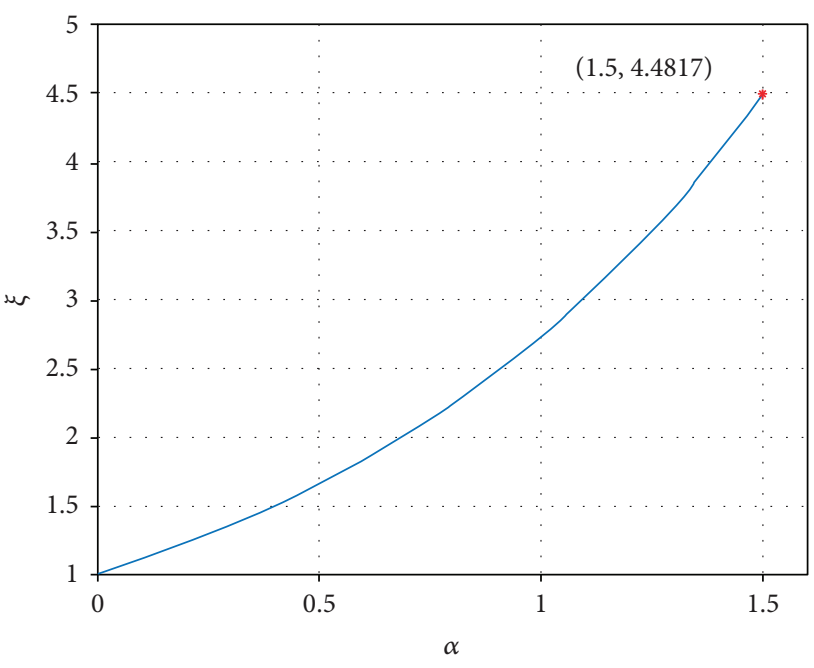

Figure 1: $\xi$ versus $\alpha$.

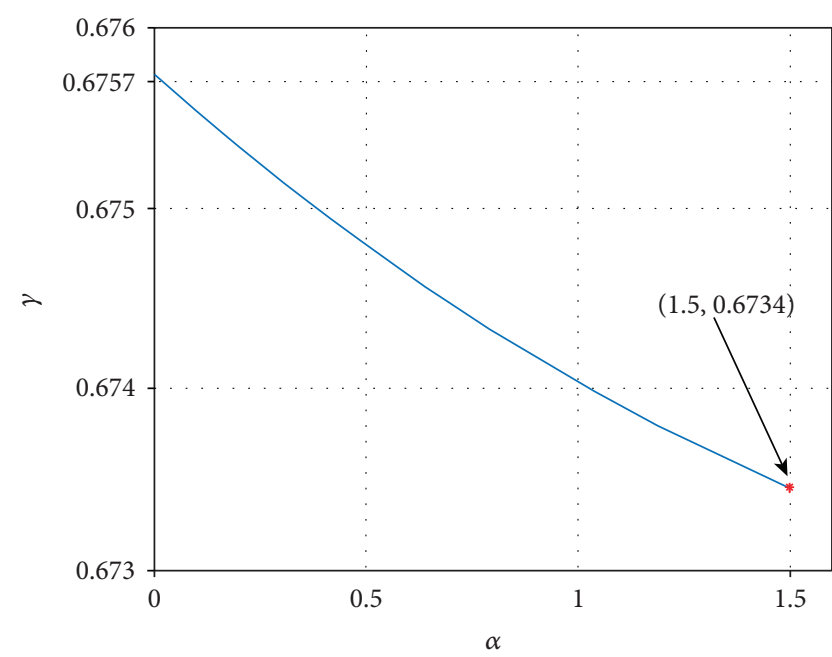

Figure 2: $\gamma$ versus $\alpha$.

0.6757 when $\alpha=0$, and the minimum value of $\gamma$ is 0.6734 when $\alpha=1.5$. The range of $\alpha$ is $[0,1.5]$ and the range of $\gamma$ is $[1,4.4817]$.

In fact, Figures 1 and 2 imply Figure 3 . To show the relations between $\gamma$ and $\xi$ clearly, Figure 3 is also drawn. From Figure 3, it can be seen that the bigger the number of $\xi$, the

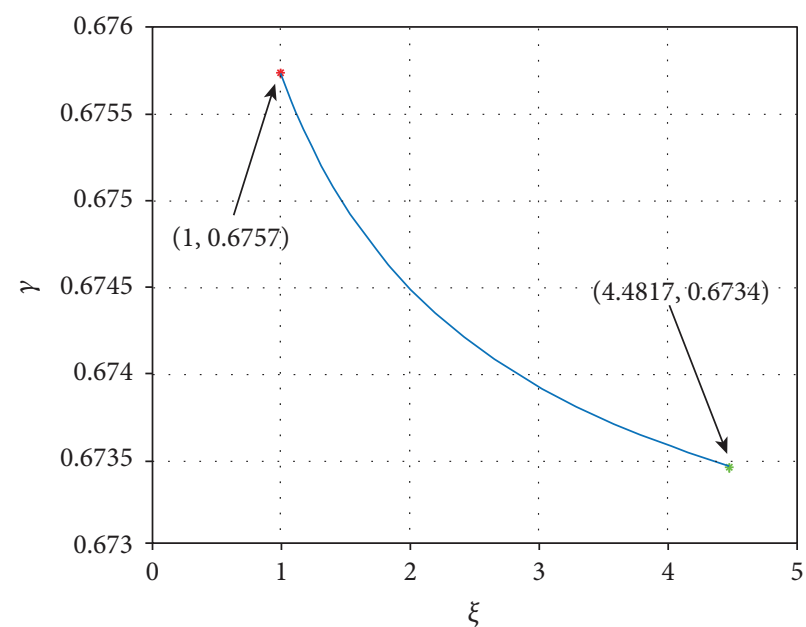

FIGURE 3: $\gamma$ versus $\xi$.

smaller $\gamma$ is. Figures 1, 2, and 3 can provide us a method for how to select the suitable SFFT $\mathrm{H}_{2} / \mathrm{H}_{\mathrm{\infty}}$ controller. If we are mainly concerned with the $\mathrm{H}_{2}$ performance, a smaller $\alpha$ can be selected. If we are mainly concerned with the $H_{\infty}$ performance, a bigger $\alpha$ can be selected.

Next, substituting $\alpha=0$ into Theorem 2 and then solving (45), (46), (47), and (48), we have

$$
\begin{aligned}
M & =\left[\begin{array}{ll}
0.6528 & 0.0810 \\
0.0810 & 0.6935
\end{array}\right], \\
Y & =\left[\begin{array}{ll}
0.0746 & -0.0635 \\
0.5236 & 0.3750
\end{array}\right], \\
\lambda & =0.3980 .
\end{aligned}
$$

Therefore, we get the following controller gain matrix:

$$
K=Y M^{-1}=\left[\begin{array}{cc}
0.1275 & -0.1064 \\
0.7460 & 0.4536
\end{array}\right] \text {. }
$$

Suppose $\quad v(t)=\sin t\left(\int_{0}^{1} \sin ^{2} t d t=0.275<h=0.5\right) \quad$ uses the Euler-Maruyama method to simulate the standard Brownian motion, one can obtain the simulated curves 


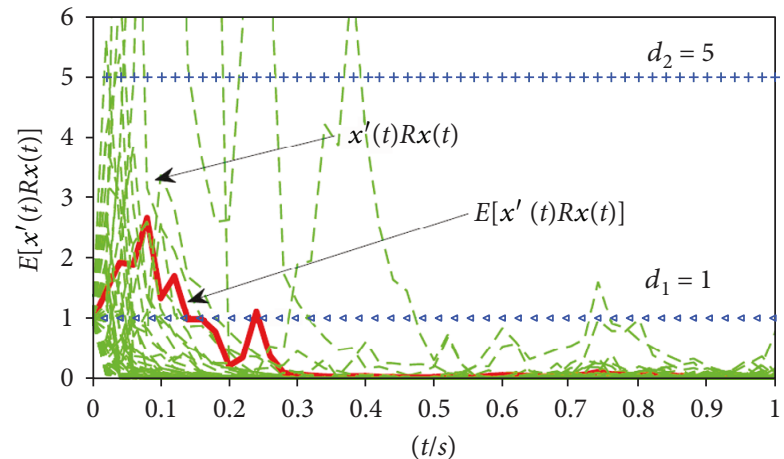

Figure 4: The response for $E\left[x^{\prime}(t) R x(t)\right]$.

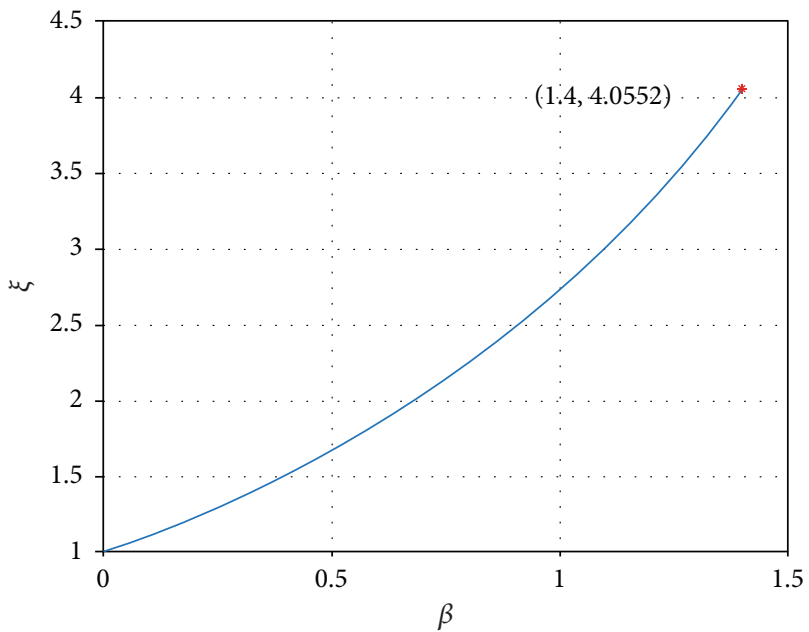

Figure 5: $\xi$ versus $\beta$.

of $x^{\prime}(t) R x(t)$ (30 curves) and $E\left[x^{\prime}(t) R x(t)\right]$ of system (10) in Figure 4. From Figure 4, we see that system (10) is FT stochastically bounded wrt $(1,5,1, I, 0.5)$.

6.2. OBFT $\mathrm{H}_{2} / \mathrm{H}_{\infty}$ Controller Design. On the basis of the SF case, Algorithm 1 can also be used to solve the similar OP for the OBFT $H_{2} / H_{\infty}$ control. The relations of $\beta$ and $\xi$ (Figure 5), $\beta$ and $\gamma$ (Figure 6), and $\gamma$ and $\xi$ (Figure 7) are obtained. From Figure 5, it can be seen that the bigger the number of $\beta$, the bigger $\xi$ is. Also, the minimum value of the $\mathrm{H}_{2}$ performance index is $\xi=1$ when $\beta=0$, and the maximum value of the $H_{2}$ performance index is $\xi=4.0552$ when $\beta=1.4$. The range of $\alpha$ is $[0,1.4]$, and the range of $\xi$ is $[1,4.0552]$.

From Figure 6, it can be seen that the bigger the number of $\beta$, the smaller $\gamma$ is. Also, the maximum value of $\gamma$ is 0.8656 when $\beta=0$, and the minimum value of $\gamma$ is 0.8413 when $\beta=1.4$. The range of $\beta$ is $[0,1.4]$, and the range of $\gamma$ is $[0.8413,0.8656]$.

In fact, Figures 5 and 6 also imply Figure 7. To show the relations between $\gamma$ and $\xi$ clearly, Figure 7 is also drawn. From Figure 7 , it can be seen that the bigger the number of

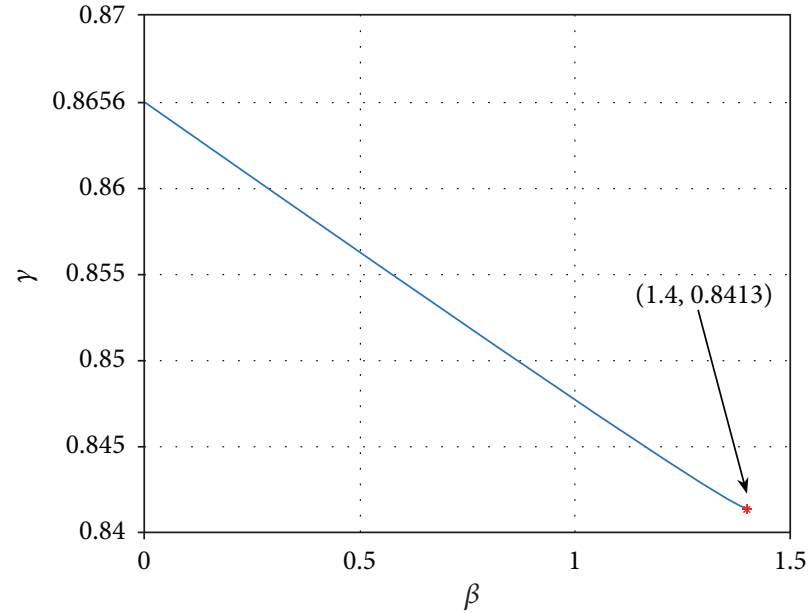

Figure 6: $\gamma$ versus $\beta$.

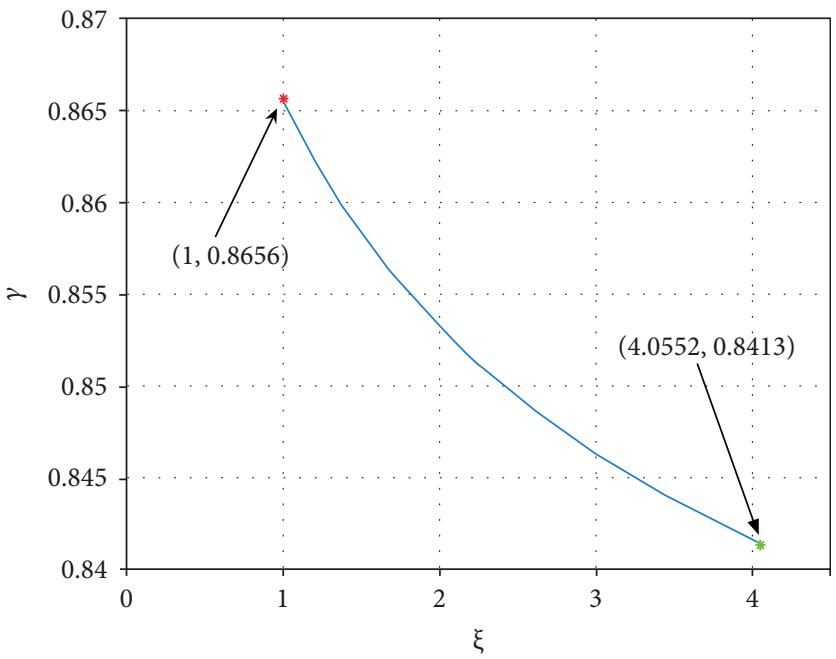

Figure 7: $\gamma$ versus $\xi$.

$\xi$, the smaller $\gamma$ is. Figures 5-7 can provide us a method for how to select the suitable OBFT $\mathrm{H}_{2} / \mathrm{H}_{\infty}$ controller. If we are mainly concerned with the $H_{2}$ performance, a smaller $\beta$ can be selected. If we are mainly concerned with the $H_{\infty}$ performance, a bigger $\beta$ can be selected.

Substituting $\beta=0$ into Theorem 4 and solving (79), (80), (81), and (82) yields

$$
\begin{aligned}
Q_{11} & =\left[\begin{array}{cc}
1.9421 & -0.0442 \\
-0.0442 & 2.2252
\end{array}\right], \\
Q_{22} & =\left[\begin{array}{ll}
3.1913 & 0.0900 \\
0.0900 & 2.2214
\end{array}\right], \\
N & =\left[\begin{array}{ll}
4.2749 & -2.9218 \\
1.6587 & 1.1962
\end{array}\right], \\
\eta & =3.6737
\end{aligned}
$$




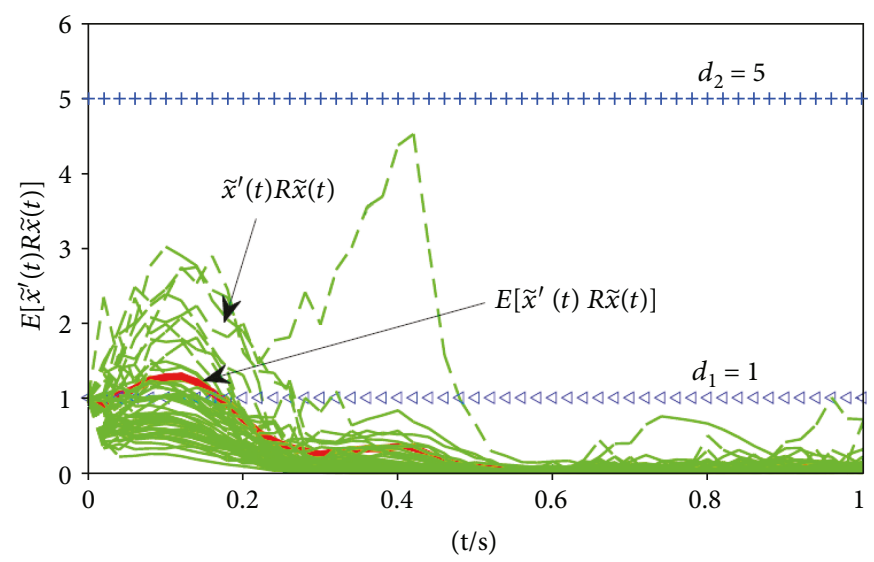

Figure 8: The response for $E\left[\tilde{x}^{\prime}(t) R \tilde{x}(t)\right]$.

Then, the corresponding observer gain matrix is obtained:

$$
L=Q_{22}^{-1} N=\left[\begin{array}{cc}
1.3200 & -0.9318 \\
0.6932 & 0.5762
\end{array}\right]
$$

Suppose $v(t)=\sin t\left(\int_{0}^{1} \sin ^{2} t d t=0.275<h\right)$ and uses the Euler-Maruyama method to simulate the standard Brownian motion, one can obtain the simulated curves of $\tilde{x}^{\prime}(t) R \tilde{x}(t)$ (30 curves) and $E\left[\tilde{x}^{\prime}(t) R \tilde{x}(t)\right]$ of system (50) in Figure 8. From Figure 8, we see that system (10) is FT stochastically bounded wrt $(1,5,1, I, 0.5)$.

\section{Conclusions}

In this study, state-feedback and observer-based finite-time $\mathrm{H}_{2} / \mathrm{H}_{\infty}$ control problems have been investigated. The problems have been transformed into optimization problems with the constraint of matrix inequalities, and a detailed optimization algorithm has been given to obtain the minimum values of the $\mathrm{H}_{2}$ index and $\mathrm{H}_{\infty}$ index. An example has been given to demonstrate the results obtained.

\section{Data Availability}

The data used to support the findings of this study are available from the corresponding author upon request.

\section{Conflicts of Interest}

The authors declare that there is no conflict of interests regarding the publication of this paper.

\section{Acknowledgments}

This work was supported by the National Natural Science Foundation of China (Grant nos. 61877062 and 61403221), China Postdoctoral Science Foundation (Grant no. 2017M610425), Natural Science Foundation of Shandong Province (Grant no. ZR2014FM019), and Open Foundation of Key Laboratory of Pulp and Paper Science and Technology of Ministry of Education of China (Grant no. KF201419).

\section{References}

[1] C. F. Wu, B. S. Chen, and W. Zhang, "Multiobjective investment policy for a nonlinear stochastic financial system: a fuzzy approach," IEEE Transactions on Fuzzy Systems, vol. 25, no. 2, pp. 460-474, 2017.

[2] R. Y. Yu and S. Gao, "Applications of probabilistic production simulation in power system," Power System Protection and Control, vol. 40, no. 11, pp. 149-155, 2012.

[3] S. Peng and F. Deng, "New criteria on pth moment inputto-state stability of impulsive stochastic delayed differential systems," IEEE Transactions on Automatic Control, vol. 62, no. 7, pp. 3573-3579, 2017.

[4] G. Chen, Y. Gao, and S. Zhu, "Finite-time dissipative control for stochastic interval systems with time-delay and Markovian switching," Applied Mathematics and Computation, vol. 310, pp. 169-181, 2017.

[5] F. Cacace, V. Cusimano, A. Germani, and P. Palumbo, "A state predictor for continuous-time stochastic systems," Systems and Control Letters, vol. 98, pp. 37-43, 2016.

[6] X. Li, X. Lin, and Y. Lin, "Lyapunov-type conditions and stochastic differential equations driven by G-Brownian motion," Journal of Mathematical Analysis and Applications, vol. 439, no. 1, pp. 235-255, 2016.

[7] Y. Wei, J. Qiu, H. K. Lam, and L. Wu, “Approaches to T-S fuzzy-affine-model-based reliable output feedback control for nonlinear Itô stochastic systems," IEEE Transactions on Fuzzy Systems, vol. 25, no. 3, pp. 569-583, 2017.

[8] W. Li, L. Liu, and G. Feng, "Distributed containment tracking of multiple stochastic nonlinear systems," Automatica, vol. 69, pp. 214-221, 2016.

[9] B. S. Chen, C. L. Tsai, and D. S. Chen, "Robust $\mathrm{H}_{\infty}$ and mixed $\mathrm{H}_{2} / \mathrm{H}_{\infty}$ filter for equalization designs of nonlinear communication systems: fuzzy interpolation approach," IEEE Transactions on Fuzzy Systems, vol. 11, no. 3, pp. 384-398, 2003.

[10] C. H. Wu, W. Zhang, and B. S. Chen, "Multiobjective $\mathrm{H}_{2} /$ $H_{\infty}$ synthetic gene network design based on promoter libraries," Mathematical Biosciences, vol. 233, no. 2, pp. 111-125, 2011. 
[11] B. S. Chen and W. Zhang, "Stochastic $\mathrm{H}_{2} / \mathrm{H}_{\infty}$ control with state-dependent noise," IEEE Transactions on Automatic Control, vol. 49, no. 1, pp. 45-57, 2004.

[12] Y. Xia, P. Shi, G. Liu, and D. Rees, "Robust mixed $\mathrm{H}_{2} / \mathrm{H}_{\infty}$ state-feedback control for continuous-time descriptor systems with parameter uncertainties," Circuits, Systems and Signal Processing, vol. 24, no. 4, pp. 431-443, 2005.

[13] Z. Yan, G. Zhang, and J. Wang, "Infinite horizon H-two/Hinfinity control for descriptor systems: Nash game approach," Journal of Control Theory and Applications, vol. 10, no. 2, pp. 159-165, 2012.

[14] H. Ma, W. Zhang, and T. Hou, "Infinite horizon $H_{2} / H_{\infty}$ control for discrete-time time-varying Markov jump systems with multiplicative noise," Automatica, vol. 48, no. 7, pp. 14471454, 2012.

[15] W. Zhang, L. Xie, and B. S. Chen, Stochastic $H_{2} / H_{\infty}$ Control: A Nash Game Approach, CRC Press, Boca Raton, FL, USA, 2017.

[16] P. M. Anderson and A. A. Fouad, Power System Control and Stability, Wiley-IEEE Press, 2nd edition, 2002.

[17] G. Kamenkov, "On stability of motion over a finite interval of time," Journal of Applied Mathematics and Mechanics, vol. 17, pp. 529-540, 1953.

[18] A. Lebedev, "The problem of stability in a finite interval of time," Journal of Applied Mathematics and Mechanics, vol. 18, pp. 75-94, 1954.

[19] A. Lebedev, "On stability of motion during a given interval of time," Journal of Applied Mathematics and Mechanics, vol. 18, pp. 139-148, 1954.

[20] F. Amato, M. Ariola, and P. Dorato, "Finite-time control of linear systems subject to parametric uncertainties and disturbances," Automatica, vol. 37, no. 9, pp. 1459-1463, 2001.

[21] Y. Dong, W. Liu, T. Li, and S. Liang, "Finite-time boundedness analysis and $H_{\infty}$ control for switched neutral systems with mixed time-varying delays," Journal of the Franklin Institute, vol. 354, no. 2, pp. 787-811, 2017.

[22] J. Cheng, H. Xiang, H. Wang, Z. Liu, and L. Hou, "Finite-time stochastic contractive boundedness of Markovian jump systems subject to input constraints," ISA Transactions, vol. 60, pp. 74-81, 2016.

[23] S. He and F. Liu, "Stochastic finite-time stabilization for uncertain jump systems via state feedback," Journal of Dynamic Systems, Measurement, and Control, vol. 132, no. 3, article 034504, 2010.

[24] S. He, Q. Ai, C. Ren, J. Dong, and F. Liu, "Finite-time resilient controller design of a class of uncertain nonlinear systems with time-delays under asynchronous switching," IEEE Transactions on Systems, Man, and Cybernetics: Systems, pp. 1-6, 2018.

[25] S. He, J. Song, and F. Liu, "Robust finite-time bounded controller design of time-delay conic nonlinear systems using sliding mode control strategy," IEEE Transactions on Systems, Man, and Cybernetics: Systems, pp. 1-11, 2018.

[26] Z. Yan, Y. Song, and J. H. Park, "Quantitative mean square exponential stability and stabilization of stochastic systems with Markovian switching," Journal of the Franklin Institute, vol. 355, no. 8, pp. 3438-3454, 2018.

[27] Z. Yan, G. Zhang, and W. Zhang, "Finite-time stability and stabilization of linear Itô stochastic systems with state and control dependent noise," Asian Journal of Control, vol. 15, no. 1, pp. 270-281, 2013.

[28] Z. Yan, W. Zhang, and G. Zhang, "Finite-time stability and stabilization of Itô stochastic systems with Markovian switching: mode-dependent parameter approach," IEEE Transactions on Automatic Control, vol. 60, no. 9, pp. 2428-2433, 2015.

[29] H. Gao, J. Xia, G. Zhuang, Z. Wang, and Q. Sun, "Nonfragile finite-time extended dissipative control for a class of uncertain switched neutral systems," Complexity, vol. 2017, Article ID 6581308, 22 pages, 2017.

[30] Z. Yan, Y. Song, and X. Liu, "Finite-time stability and stabilization for Itô-type stochastic Markovian jump systems with generally uncertain transition rates," Applied Mathematics and Computation, vol. 321, pp. 512-525, 2018.

[31] Y. Zhang, P. Shi, S. K. Nguang, and H. R. Karimi, "Observerbased finite-time fuzzy $H_{\infty}$ control for discrete-time systems with stochastic jumps and time-delays," Signal Processing, vol. 97, pp. 252-261, 2014.

[32] Y. Zhang, P. Shi, S. K. Nguang, and Y. Song, "Robust finitetime $H_{\infty}$ control for uncertain discrete-time singular systems with Markovian jumps," IET Control Theory \& Applications, vol. 8, no. 12, pp. 1105-1111, 2014.

[33] Y. Zhang, P. Shi, and S. K. Nguang, "Observer-based finitetime $H_{\infty}$ control for discrete singular stochastic systems," Applied Mathematics Letters, vol. 38, pp. 115-121, 2014.

[34] F. B. Hanson, Applied Stochastic Processes and Control for Jump-Diffusions: Modelling, Analysis and Computation, SIAM, Philadelphia, PA, USA, 2007. 


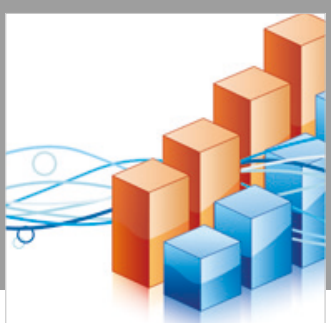

Advances in

Operations Research

\section{-n-m}
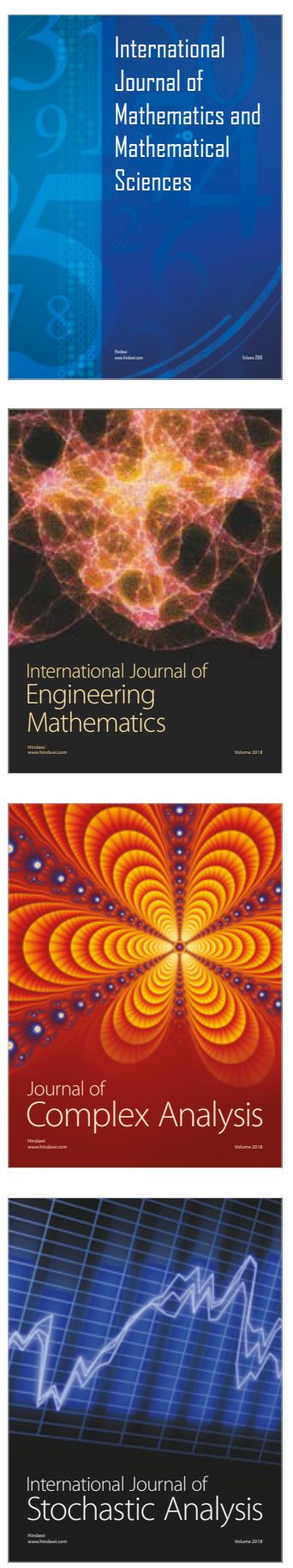
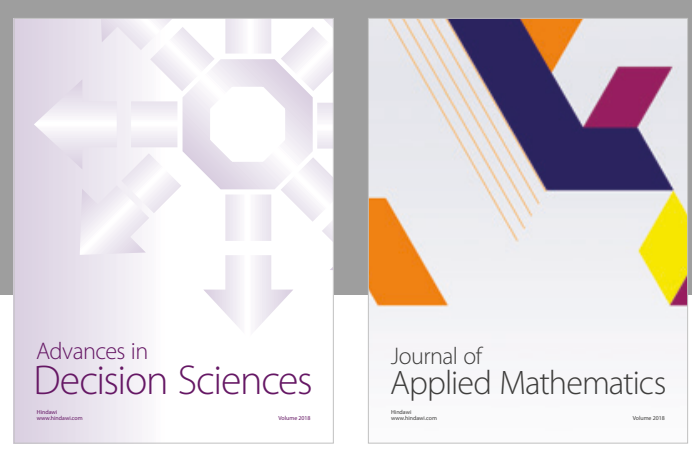

Journal of

Applied Mathematics
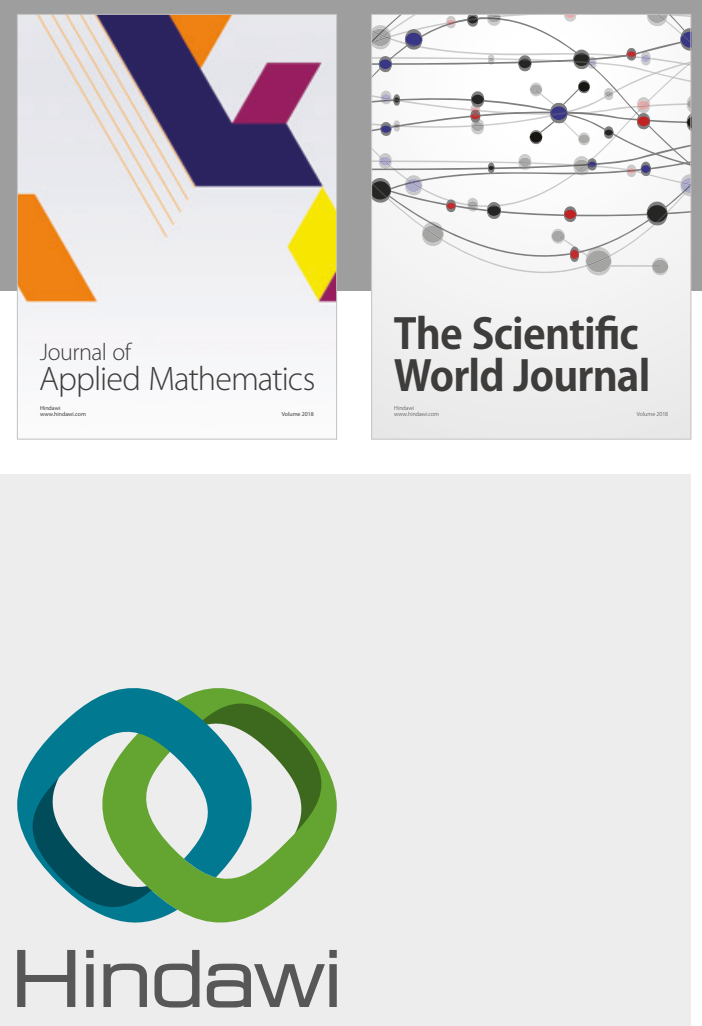

Submit your manuscripts at

www.hindawi.com

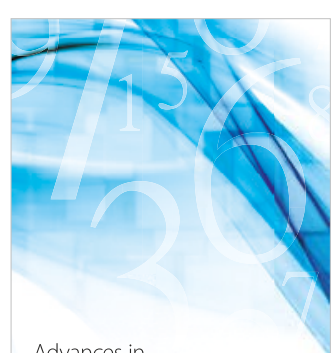

Advances in
Numerical Analysis
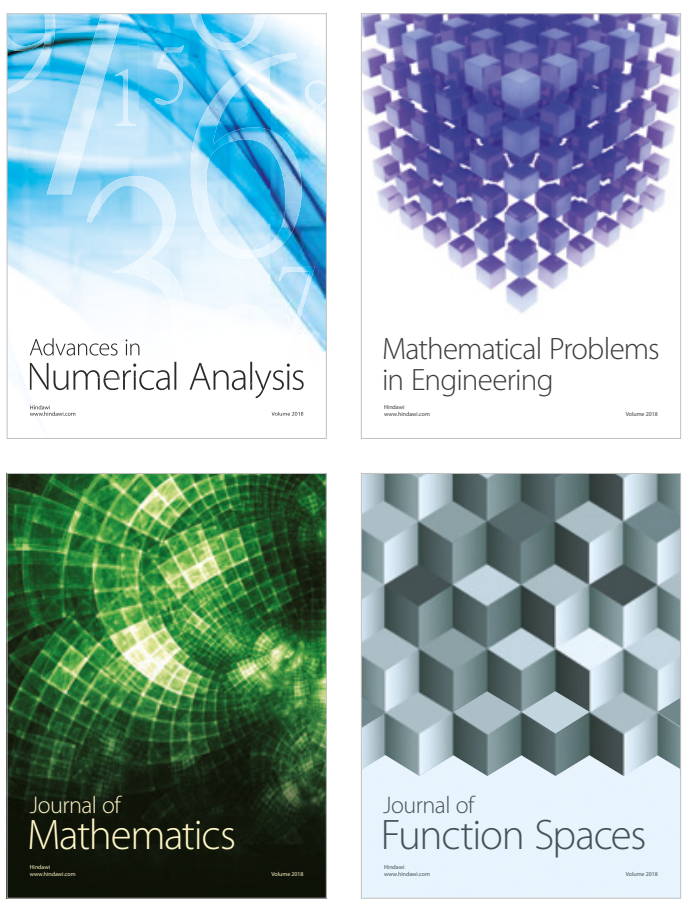

Mathematical Problems in Engineering

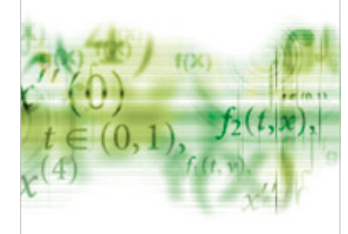

International Journal of

Differential Equations

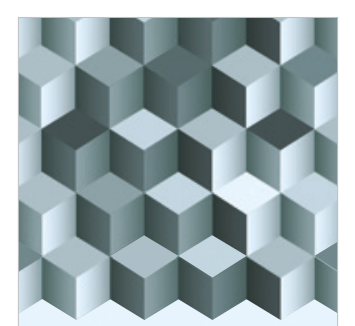

Journal of

Function Spaces
The Scientific

World Journal

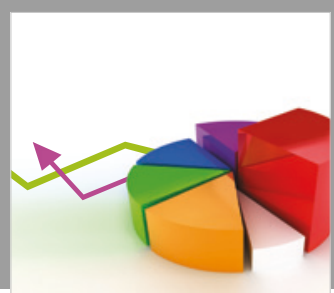

Journal of

Probability and Statistics
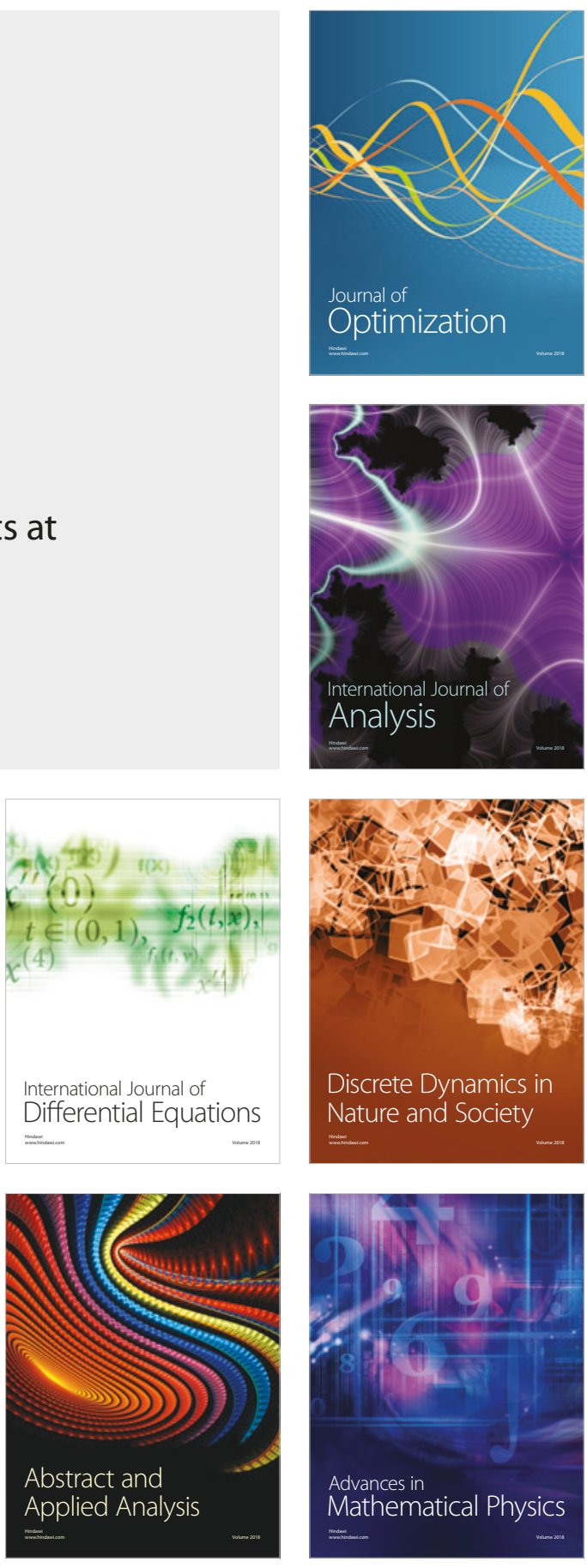